\title{
Chiral Recognition with Room-Temperature Phosphorescence in Guest-Host Energy-Transfer Systems
}

\author{
Biao Chen, ${ }^{*[a]}$ Wenhuan Huang, ${ }^{[a]}$ and Guoqing Zhang ${ }^{*[a]}$ \\ [a] Prof. B. Chen, Dr. W. Huang, Prof. G. Zhang \\ Hefei National Laboratory for Physical Science at the Microscale, \\ University of Science and Technology of China, \\ 96 Jinzhai Rd, Hefei, Anhui 230026 (China) \\ E-mail: gzhang@ustc.edu.cn; biaochen@ustc.edu.cn. \\ Supporting information for this article is given via a link at the end of the document.
}

\begin{abstract}
Chiral recognition with molecular luminescence is a beneficial but challenging method due to an often lack of dramatic difference in intermolecular interactions between the chiral analyte/substrate pair versus their enantiomeric counterpart. Here we show that the difference in room-temperature phosphorescence (RTP) can be substantially augmented by employing guest-host energy-transfer systems. By covalent attachment of chiral amino compounds to a phthalimide host and a naphthalimide guest, respectively, RTP intensity difference of two orders of magnitude with a detection limit of $>98 \%$ enantiomeric excess (ee) could be achieved. For example, S-enantiomeric guests in S-enantiomeric hosts produce strong red RTP afterglow while no appreciable RTP could be observed in an S/R guest-host combination. The huge spectroscopic difference in RTP results in conspicuous steady-state and delayed-emission variations, which could easily be discriminated by the naked eye. A generalized concept in solid-state RTP chiral recognition is proposed to expand the application scope of the reported method.
\end{abstract}

Chiral recognition ${ }^{[1]}$ is vital in pharmaceutical ${ }^{[2]}$ and life sciences, as is often the case where for a drug ${ }^{[3]}$ one enantiomer cures while the mirror-image other kills. ${ }^{[4]}$ Many techniques, including nuclear magnetic resonance (NMR), circular dichroism (CD), gas chromatography (GC), mass spectrometry (MS), and high-performance liquid chromatography (HPLC), have been developed to differentiate these isomers. Among these methods, photoluminescence-based enantiomeric discrimination ${ }^{[5]}$ is of interests in the past several decades as it is real-time, low-cost, and highly sensitive. Many luminescence chiral selectors based on 1,1'-bi-2-naphthol, ${ }^{[6]}$ tetraphenylethene, ${ }^{[7]}$ and polymers ${ }^{[8]}$ have been reported, with sensing modalities ranging from emission intensity to lifetime, and to color..$^{\left[{ }^{[]}\right.}$Room-temperature phosphorescence (RTP), on the other hand, exhibits extra benefits, such as red-shifted wavelengths into the visible region and longer lifetimes ${ }^{[10]}$ for enantiomeric discrimination with higher sensitivity. ${ }^{[11]}$ RTP chiral selectors are usually rigid media such as inclusion complexes (e.g., cyclodextrin ${ }^{[12]}$ ) or solid-state substrates ${ }^{[13]}$ (e.g., menthol) which are resistant to diffusing molecular oxygen and other quenchers, and the signal distinction, albeit scarce in examples, is subtle with only minor differences showing up in lifetimes between the two enantiomeric phosphors. The subtlety is not surprising since such a discrimination relies solely on the limited environmental disparity effects, which mainly dictate the difference in interactions between chemical groups of the analyte and substrate. Consequently, the key to success in RTP chiral recognition is to amplify such a disparity. Herein, we report a new RTP chiral recognition method by using an organic guesthost system consisting of a brominated phthalimide host and an amino naphthalimide guest (Scheme 1), where the RTP intensity can be expressed by the following relationship: $I_{\mathrm{RTP}} \propto \eta_{\mathrm{ET}} \cdot \mathrm{k}_{\mathrm{ISC}}$. $1 / k_{n r}$, where $\eta_{E T}$ is the efficiency of excited-state energy transfer, $\mathrm{k}_{\mathrm{ISC}}$ is the rate of intersystem crossing, and $\mathrm{k}_{\mathrm{nr}}$ is the nonradiative decay rate. As Scheme 1 indicates, we expect that all three variables are related to chirality (e.g., spin polarization and orbital overlap during energy transfer) or chirality-related secondary effects (e.g., $k_{n r}$ and $k_{\text {Isc }}$ difference from presence or absence in close molecular contacts with external heavy-atom effects), so that amplification of the RTP intensity difference could be significant enough for the naked eye.

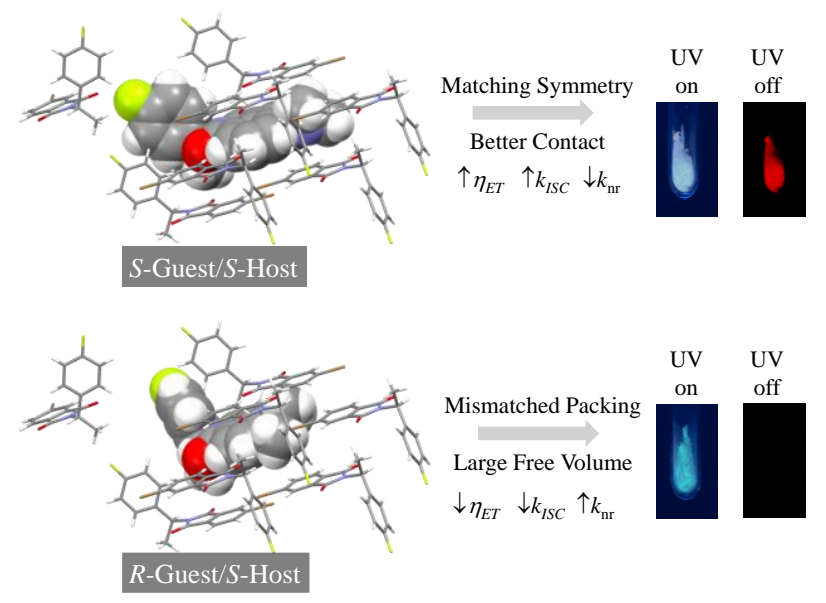

Scheme 1. Illustration of matching and mismatching molecular packing arrangements in guest-host systems of the same and different chirality, which potentially impact the RTP yield related to the efficiency of excited-state energy transfer $\eta_{E T}$, the rate of intersystem crossing $k_{I S C}$, and the non-radiative decay rate $\mathrm{k}_{\mathrm{n}}$.

Two chiral amino compounds are chemically modified into naphthalimides $\boldsymbol{R}$-4FMNNI and $\boldsymbol{S}$-4FMNNI (Figure 1a), as guest molecules, and two host ones $(\boldsymbol{R}-\mathbf{4 F B r B I}$ and $\mathbf{S}-4 \mathrm{FBrBI}$, Figure 1b), which are characterized by ${ }^{1} \mathrm{H}$ and ${ }^{13} \mathrm{C}$ NMR spectra, high resolution mass spectrometry (HRMS, Figure S32-49), elemental analysis (EA) and chiral high-performance liquid chromatography (CHPLC). The CHPLC traces (Figure S1-S2, Table S1-S2) demonstrate that the model compounds are chiral molecules with high purity $>99.5 \%$ with ee values $>99.9 \%$. Crystal parameters (Figure 1c) obtained from single-crystal $X$ ray diffraction (SC-XRD) reveal that these molecules exhibit the same monoclinic system, space group $\left(P 2_{1}\right)$, and similar dihedral angles between the phenyl and imide planes (109.28 $\left.113.32^{\circ}\right)$, suggesting high structural similarity in the solid state. The CD spectra (Figure S6) are used to determine their chirality 
with absolute CD signal of 10-20 mdeg $(0.1 \mathrm{mM})$. The absorption (Figure S5) and emission spectra (Figure S7) in dilute dichloromethane $\left(\mathrm{CH}_{2} \mathrm{Cl}_{2}\right)$ show that 4FMNNI set exhibit intense (Figure S7, Table S5) fluorescence while 4FBrBI have no discernible emission, likely due to the heavy-atom effect. ${ }^{[14]} \mathrm{At}$ $77 \mathrm{~K}, 4 \mathrm{FBrBI}$ displays phosphorescence with a lifetime of several milliseconds, while 4FMNNI exhibits no appreciable delayed emission (Figure S9), suggesting its low intrinsic phosphorescence yield. ${ }^{[15]}$ a)

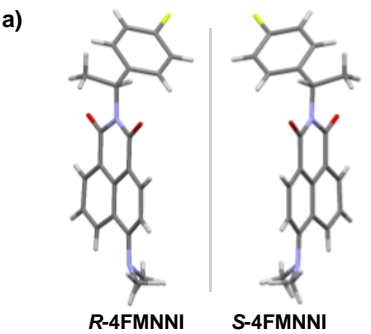

c)

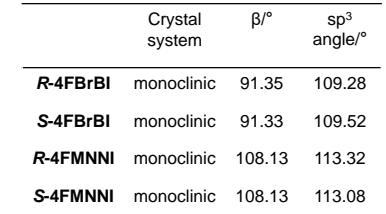

e)

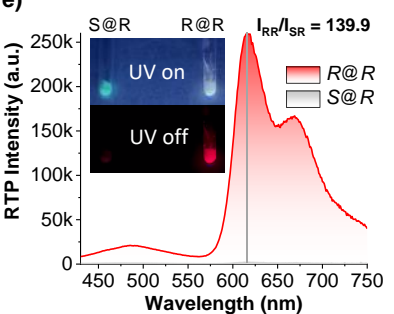

b)

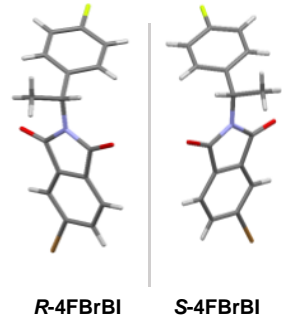

d)

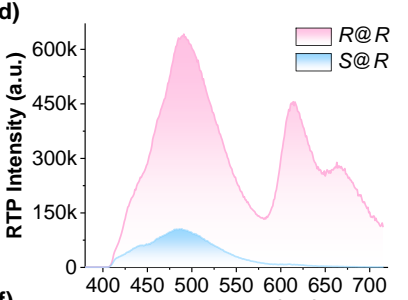

Wavelength $(\mathrm{nm})$

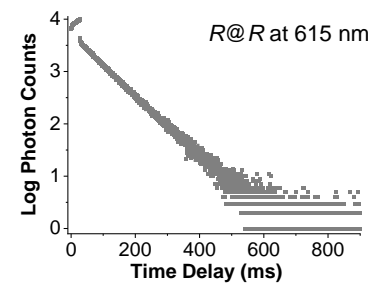

Figure 1. RTP Chiral recognition with ef $>10^{2}$ : a) Molecular configurations obtained from single-crystal X-ray diffraction (SC-XRD) for analyte guests $\boldsymbol{R}$ 4FMNNI and S-4FMNNI. b) SC-XRD structures for chiral selector hosts $\boldsymbol{R}$ 4FBrBI and S-4FBrBI. c) Crystal lattice parameters with $\alpha\left(90^{\circ}\right)$ and $\gamma\left(90^{\circ}\right)$ omitted. d) Steady-state photoluminescence (PL) spectra of two chiral guests (w/w 10 ppm, pink for $\boldsymbol{R}$-4FMNNI and light blue for $\boldsymbol{S}$-4FMNNI) in the $\boldsymbol{R}$ 4FBrBI chiral selector host solid at $298 \mathrm{~K}\left(\lambda_{\mathrm{ex}}=365 \mathrm{~nm}\right)$. e) Delayed emission (DE, $\Delta t=0.1 \mathrm{~ms}$ ) spectra of two chiral guests (w/w $10 \mathrm{ppm}$ ) in the $\boldsymbol{R}-\mathbf{4} \mathrm{FBrBI}$ solid at $298 \mathrm{~K}\left(\lambda_{\mathrm{ex}}=424 \mathrm{~nm}\right)$. Inset: photographs of combinations of two guests in $\boldsymbol{R}-\mathbf{4} \mathrm{FBrBI}$ during and immediately after $365-\mathrm{nm}$ light irradiation. f) Time-resolved RTP emission at $615 \mathrm{~nm}$ for $\boldsymbol{R}$-4FMNNI in solid $\boldsymbol{R}-\mathbf{4 F B r B I}$ at a concentration of $10 \mathrm{ppm}$.

The chiral selector $\boldsymbol{R}$-4FBrBI was first examined as a solidstate host: cyan fluorescence (circa $491 \mathrm{~nm}, \tau=3.62 \mathrm{~ns}$ ) and red RTP (circa $615 \mathrm{~nm}$ ) arise simultaneously constituting a visibly white emission color when $\boldsymbol{R}-\mathbf{4 F M N N I}(10 \mathrm{ppm}$, w/w, see $\mathrm{SI}$ for detailed protocols) is mixed with the selector medium (R@R, Figure 1d). Surprisingly, when its enantiomer ( $S$ 4FMNNI) is applied to the $\boldsymbol{R}-\mathbf{4 F B r B I}$ selector, only fluorescence occurs with no distinguishable RTP. Delayed emissions (DE) show that the enantiomeric RTP enhancement ratio $\left(\right.$ ef $\left.=I_{R R} / I_{\mathrm{SR}}\right)$ is 139.9 (Figure 1e), with an R@R RTP lifetime of $73.31 \mathrm{~ms}$ (Figure 1d, Table 1). Subtraction of the background host signal from the total RTP gives an ef $f_{\Delta}\left(\Delta I_{R R} / \Delta I_{S R}=\left[I_{R R^{-}} I_{R}\right] /\left[I_{S R}-I_{R}\right]\right)$ of 196.6 (Figure S10). The achieved RTP chiral recognition via organic chiral solids (OSC), which is essentially an "on-off" mode.
DE spectra excited with 365-nm light display lower ef values due to host RTP interference (Figure S10 and S12-14). The selector host $\mathbf{S}-\mathbf{4} \mathbf{F B r B I}$, the enantiomer of $\boldsymbol{R}-\mathbf{4} \mathbf{F B r B I}$, can also achieve enantioselectivity as shown in Figure $\mathrm{S} 11$, where a "mirrorimage relation" of RTP responses is observed toward the enantiomers of the 4FMNNI, i.e., ef $\left(\mathrm{I}_{\mathrm{SS}} / \mathrm{I}_{\mathrm{RS}}\right)=155.3$ with an RTP lifetime of $70.55 \mathrm{~ms}$ for S@S confirms the consistency of the chiral recognition method using the guest-host system.

Table 1. Photoluminescence properties of dopant samples (w/w $10 \mathrm{ppm}$ ) and pure host solids at room temperature.

\begin{tabular}{cccccc}
\hline Samples[a] $^{[\mathrm{F}}$ & $\begin{array}{c}\mathrm{F} \max \\
(\mathrm{nm})^{[\mathrm{b}]}\end{array}$ & $\begin{array}{c}\text { Lifetime } \\
(\mathrm{ns})^{[\mathrm{c}]}\end{array}$ & $\begin{array}{c}\mathrm{P} \max \\
(\mathrm{nm})^{[\mathrm{d}]}\end{array}$ & $\begin{array}{c}\text { Lifetime } \\
(\mathrm{ms})^{[\mathrm{e}]}\end{array}$ & $\mathrm{e}^{[\mathrm{f}]}$ \\
$\mathbf{R} @ \mathbf{R}$ & 491 & 3.62 & 615 & 73.31 & 139.9 \\
$\mathbf{S} @ \mathbf{R}$ & 489 & 6.22 & 618 & 44.79 & \\
$\mathbf{S} @ \mathbf{S}$ & 481 & 5.41 & 617 & 70.55 & 155.3 \\
$\mathbf{R} @ \mathbf{S}$ & 483 & 5.50 & 618 & 41.94 & \\
$\mathbf{R - 4 F B r B I}$ & $/[\mathrm{g}]$ & $/$ & 482 & 0.62 & $/$ \\
$\mathbf{S - 4 F B r B I}$ & $/$ & $/$ & 482 & 0.85 & $/$ \\
\hline
\end{tabular}

[a] Guest-host molecular solids (w/w $10 \mathrm{ppm}$ ). [b] Fluorescence emission maxima of steady-state photoluminescence spectra excited at $365 \mathrm{~nm}$. [c] Apparent fluorescence lifetime (Weighted average sum, naonoLED-370). [d] RTP maxima of delayed emission spectra excited at $424 \mathrm{~nm}$. [e] Apparent RTP lifetime (spectraLED-370). [f] enantiomeric RTP enhancement ratios (efRTP, Iss/lRs or IRR/lsR). [g] Not exist.

The guest/host ratio and its influences on enantioselectivity were then investigated. The PL spectra were presented in Figure 2a and Figure S17: when the host and the guest possess the same chirality (i.e., R@R or $\mathbf{S} @ \mathbf{S}$ ), dramatically stronger RTP in the wavelength range of 600-720 nm emerges compared to samples of different chiralities (S@R or $\mathbf{R} @ \mathbf{S}$ ), resulting in ef typically $>10$. The ef value of a guest-host doping of $0.1 \%$ is found to be $<3$ because of intense guest fluorescence extending to the red region and elevating the baseline. On the other hand, $\mathbf{S} @ \mathbf{R}$ (or $\mathbf{R} @ \mathbf{S}$ ) also exhibits appreciable RTP intensity when doped at high concentrations (> $100 \mathrm{ppm}$ ), possibly from guest aggregates formation ${ }^{[16]}$ or contribution from the enantiomeric impurity limited by current instrument detections. The highest ef is obtained in a doping ratio range of 1 to 100 ppm (e.g., ef $>55$ for the 10-ppm sample), which is consistent with the DE spectra (Figure 2b-2d). Furthermore, the DE spectra present higher ef values (from 24.5 to 155.3 when guest/host ratio $1 \mathrm{ppm}-100$ $\mathrm{ppm})$ than steady-state $\mathrm{PL}$ via eliminating short-lived luminescence interference (e.g., guest fluorescence and host phosphorescence), showcasing the prominent advantage from RTP chiral recognition. We found that the guest doping concentration of $1 \mathrm{ppm}$ is sufficient for an highly enantioselective RTP enhancement of 24.5-32.0 folds, while many chiral recognition methods such as enantioselective fluorescence enhancement ${ }^{[17]}$ demands that the enantiomer analyte be at least in equal proportion or dozens of times in excess to the chiral selector ${ }^{[18]}$ With its high sensitivity, the guest-host RTP recognition method could be applied to ultratrace chiral analysis. Notably, samples with an identical chirality (S@S or $\mathbf{R} @ \mathbf{R})$ present excitation-dependent PL emission (Figure S18-21): the fluorescence intensity increases while the RTP intensity barely changes with different excitation wavelengths (from $320 \mathrm{~nm}$ to 
$424 \mathrm{~nm}$ ), leading to a divergent $\mathrm{P} / \mathrm{F}$ ratio (Figure $\mathrm{S} 18-20 \mathrm{~d}$ ). Specially, in the $350-380 \mathrm{~nm}$ range, the $P / F$ ratio decreases as the guest absorbance increases (Figure S5).

a)

b)

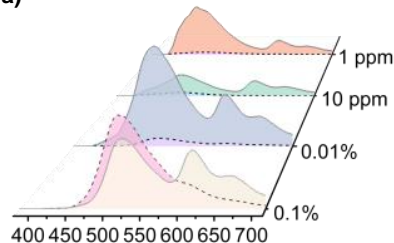
Wavelength $(\mathrm{nm})$

c)
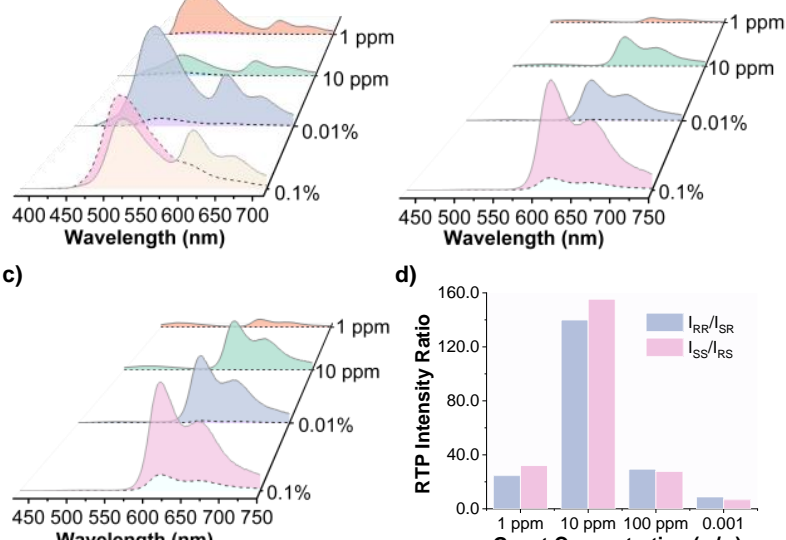

Wavelength $(\mathrm{nm})$

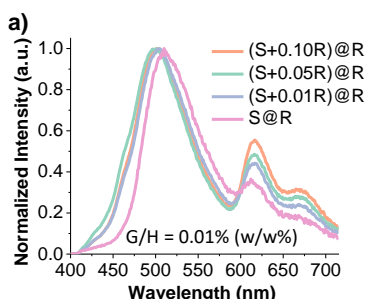

b)

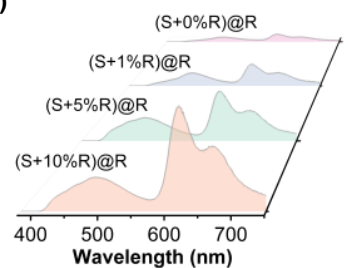

c)

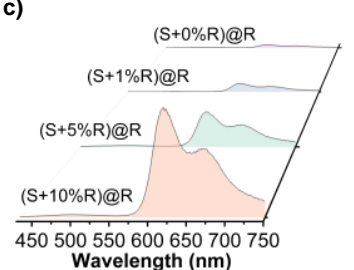

d)

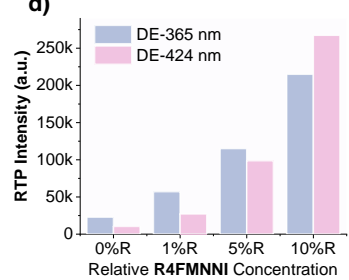

Figure 3. Enantiomer recognition with different ee values: a) Steady-state $\mathrm{PL}$ spectra of $\boldsymbol{R}$-4FMNNI dopants (0\%-10\%) in 0.01\% (100 ppm) S-4FMNNI@R4FBrBI solid at air at $298 \mathrm{~K}\left(\lambda_{\mathrm{ex}}=365 \mathrm{~nm}\right)$. Delayed emission (DE, $\Delta t=0.1 \mathrm{~ms}$ ) spectra of $\boldsymbol{R}$-4FMNNI dopants (0\%-10\%) in $0.01 \%$ S-4FMNNI@R-4FBrBI solid at air at $298 \mathrm{~K}\left(\lambda_{\mathrm{ex}}=365 \mathrm{~nm}\right.$ for b) and $424 \mathrm{~nm}$ for $\left.\mathrm{c}\right)$ ). d) Intensity of DE emission at $618 \mathrm{~nm}$ vs. Percentage of $\boldsymbol{R}-\mathbf{4 F M N N I}$ dopants ( $0 \%-10 \%)$.

Figure 2. a) Steady-state $\left(\lambda_{\mathrm{ex}}=365 \mathrm{~nm}\right)$ and b) delayed emission (DE, $\Delta t=$ $0.1 \mathrm{~ms}, \lambda_{\mathrm{ex}}=424 \mathrm{~nm}$ ) spectra of $\boldsymbol{R}$-4FMNNI (solid borderline) and S-4FMNNI (dash borderline) guests ( $\mathrm{w} / \mathrm{w}=1 \mathrm{ppm}-0.1 \%)$ in the $\boldsymbol{R}-\mathbf{4 F B r B I}$ solid chiral selector medium in air at $298 \mathrm{~K}\left(\lambda_{\mathrm{ex}}=365 \mathrm{~nm}\right)$. c) Delayed emission (DE, $\Delta t=$ $0.1 \mathrm{~ms}$ ) spectra of $\boldsymbol{R}$-4FMNNI (dash borderline) and $\mathbf{S}-\mathbf{4 F M N N I}$ (solid borderline) guests ( $w / w=1 \mathrm{ppm}-0.1 \%)$ in $\mathbf{S}-4 \mathbf{F B r B I}$ solid in air at $298 \mathrm{~K}\left(\lambda_{\text {ex }}=\right.$ $424 \mathrm{~nm}$ ). d) The ef $_{\text {RTP }}$ value (intensity of DE emission at $620 \mathrm{~nm}$ ) vs. concentation of guest dopants $(\mathrm{w} / \mathrm{w}=1 \mathrm{ppm}-0.1 \%)$.

To test the chiral differentiation potential of the system, by using $\mathbf{S}-4 \mathbf{F M N N I}$-doped $\boldsymbol{R}-\mathbf{4 F B r B I}$ (w/w $0.01 \%$ ) as an example, the RTP spectroscopic changes with $\boldsymbol{R}$-4FMNNI at varying enantiomeric composition were recorded, where $\mathrm{S}+0 \% \mathrm{R}$, $\mathrm{S}+1 \% \mathrm{R}, \mathrm{S}+5 \% \mathrm{R}$ and $\mathrm{S}+10 \% \mathrm{R}$ represent that the ee values, $([\mathrm{S}]-[\mathrm{R}]) /([\mathrm{S}]+[\mathrm{R}])$, of the partially racemic guest analyte 4FMNNI compounds are $100 \%, 98.02 \%, 90.48 \%$ and $81.82 \%$, respectively (Figure 3 and Figure S22). As can be observed, the RTP intensity is enhanced with a higher R-guest proportion. Remarkably, there is a measurable RTP improvement $(2-3 \times$ by tuning the excitation wavelength) between ee values of $100 \%$ and $98 \%$, implying the successful distinguishability for an enantiomer ( $\boldsymbol{R}$-4FMNNI in this case) lower than $1 \%$ from the racemate with the current RTP chiral recognition method. i.e., the method can be used to resolve the enantiomeric composition and an enantiomer impurity with ee $>98 \%$.

Finally, the guest-host RTP chiral recognition method was extended to a different amino compound. Chiral amino alcohols have been extensively used in medicine, racemate resolution and asymmetric synthesis. ${ }^{[19]}$ Enantioselective recognition of amino alcohols has thus attracted significant research attention in recent years. ${ }^{[20]}$ Here we show that the same RTP chiral recognition method could be applied to a chiral amino alcohol, using 2-phenylglycinol $(\mathrm{Pg})$ as an example. Following the same protocol, four chiral derivatives (Figure 4a, $\boldsymbol{R}$-PgMNNI and $\boldsymbol{S}$ PgMNNI as guest molecules) and two racemate (Figure S3-4, Figure S50-S67) were synthesized and characterized. When doped at a concentration of $100 \mathrm{ppm}, \mathrm{PL}$ spectra (Figure 4b and Figure S25 and S28) reveal that the host-guest system with the same chirality (R@R and $\mathbf{S} @ \mathbf{S})$ exhibits more pronounced RTP enhancement over $\mathbf{S} @ \mathbf{R}$ or $\mathbf{R} @ \mathbf{S}$, where even the naked eye can readily discriminate the enantioselective luminescence under UV excitation, since $\mathbf{S} @ \mathbf{R}$ (or $\mathbf{R} @ \mathbf{S}$ ) shows cyan emission while the other two are either orange or pink (photos in Figure 4). The DE spectra (Figure 4c) also register an RTP enantioselective enhancement $>10$ folds $\left(\mathrm{I}_{\mathrm{RR}} / \mathrm{I}_{\mathrm{SR}}=11.5\right.$ and $\mathrm{IsS}_{\mathrm{SS}} \mathrm{I}_{\mathrm{RS}}=12.7$ ) with a longer lifetime (Figure $4 \mathrm{~d}, 63.25 \mathrm{~ms}$ for $\mathbf{S} @ \mathbf{S}, 63.36$ ms for $\mathbf{R} @ \mathbf{R}$, Table S6). The excitation dependent PL (Figure S23-28) and DE spectra (Figure S29) are analogous to the amino fluorobenzyl compound, suggesting that a similar photophysical process may be involved for the chiral sensing scheme for both amino fluorobenzyl and amino alcohol compounds. 

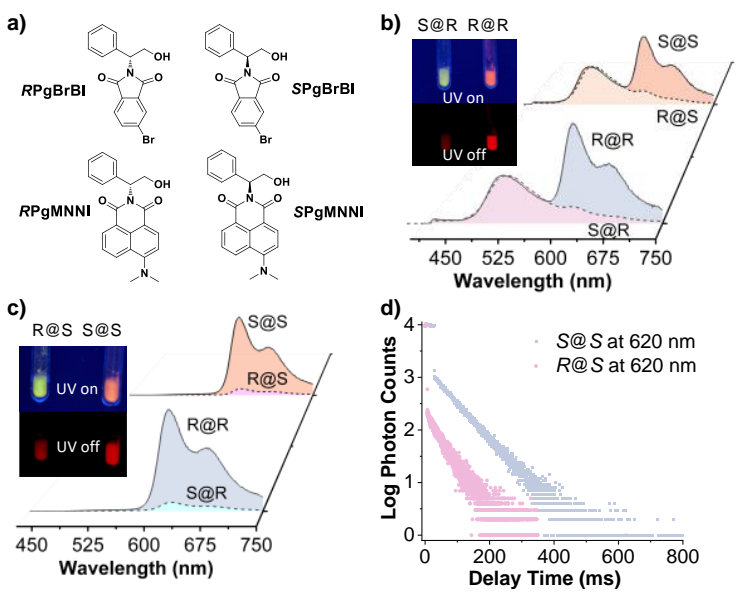

Figure 4. Chiral amino alcohol recognition: a) Molecular structures chiral amino alcohol derivatives: chemically modified chiral analyte guests $(\boldsymbol{R}$ PgMNNI and S-PgMNNI) and chiral selector hosts $(\boldsymbol{R}-\mathbf{P g B r B I}$ and $\boldsymbol{S}-\mathbf{P g B r B I})$ molecules. b) Normalized steady-state PL spectra of two chiral guests (w/w = $100 \mathrm{ppm})$ in two chiral host solids at $298 \mathrm{~K}\left(\lambda_{\mathrm{ex}}=380 \mathrm{~nm}\right)$. Inset: photographs of combinations of two guests in $\boldsymbol{R}-\mathbf{P g B r B l}$ during and immediately after 365 $\mathrm{nm}$ light irradiation. c) Delayed emission (DE, $\Delta t=0.1 \mathrm{~ms}$ ) spectra of two guests (w/w $100 \mathrm{ppm})$ in two host solids at $298 \mathrm{~K}\left(\lambda_{\mathrm{ex}}=424 \mathrm{~nm}\right)$. Inset: photographs of combinations of two guests in S-PgBrBI during and immediately after 365-nm light irradiation. d) Time-resolved emission intensity at $620 \mathrm{~nm}$ of $\boldsymbol{R}$-PgMNNI and $\mathbf{S}-\mathbf{P g M N N I}$ in the solid-state $\boldsymbol{R}$-PgBrBI

In summary, we have successfully demonstrated a photoluminescence chiral recognition method by taking advantage of the more sensitive guest-host room-temperature phosphorescence (RTP). We show that by chemically modifying chiral amino compounds into phthalimide selector hosts and naphthalimide analyte guest, an RTP intensity difference of up to 196 folds can be obtained. In combination with fluorescence emission, the dramatic chirality-dependent RTP alters the emission color under UV light, making it easily recognized by the naked eye. To show its application potential, ultratrace analyses were also performed and the method was able to resolve enantiomeric impurity in a partial racemate with an ee value > $98 \%$. Although the method is not yet general, it nevertheless gives one of the best performances in optical chiral recognition so far, and paves way for more generalized, energy-transferbased guest-host chiral recognition methodologies. The future reports will be focused on detailed mechanistic study for the amplified chirality-dependent RTP, as well as extension to other chemical modification methods for other chiral molecules.

\section{Acknowledgements}

We thank the National Natural Science Foundation (22003063 to B. C. and 21975238 to G. Z.) for financial support. We also thank Prof. Yi Luo for his helpful discussion. CCDC 21504132150416 contain the supplementary crystallographic data for this paper.

Keywords: binary doping system $\cdot$ chiral recognition $\cdot$ host and

guest $\cdot$ organic chiral solid $\cdot$ room temperature phosphorescence
[1] a) J. Hu, W. G. Cochrane, A. X. Jones, D. G. Blackmond, B. M. Paegel, Nat. Chem. 2021, 13, 786-791; b) P. Howlader, E. Zangrando, P. S. Mukherjee, J. Am. Chem. Soc. 2020, 142, 9070-9078.

[2] a) Z. Liu, X. Li, H. Masai, X. Huang, S. Tsuda, J. Terao, J. Yang, X. Guo, Sci. Adv. 2021, 7, eabe4365; b) G. Zhu, O. J. Kingsford, Y. Yi, K.y. Wong, J. Electrochem. Soc. 2019, 166, B1226-B1231.

[3] Z. Du, C. Liu, H. Song, P. Scott, Z. Liu, J. Ren, X. Qu, Chem 2020, 6 , 2060-2072.

[4] M. E. Matyskiela, S. Couto, X. Zheng, G. Lu, J. Hui, K. Stamp, C. Drew Y. Ren, M. Wang, A. Carpenter, Nat. Chem. Biol. 2018, 14, 981-987.

[5] a) M. Hu, Y.-X. Yuan, W. Wang, D.-M. Li, H.-C. Zhang, B.-X. Wu, M. Liu, Y.-S. Zheng, Nat. Commun. 2020, 11, 1-10; b) L. Pu, Acc. Chem. Res. 2017, 50, 1032-1040.

[6] X. Wu, X. Han, Q. Xu, Y. Liu, C. Yuan, S. Yang, Y. Liu, J. Jiang, Y. Cui, J. Am. Chem. Soc. 2019, 141, 7081-7089.

[7] A. Nitti, D. Pasini, Adv. Mater. 2020, 32, 1908021.

[8] S. Mishra, A. K. Mondal, E. Z. Smolinsky, R. Naaman, K. Maeda, T. Nishimura, T. Taniguchi, T. Yoshida, K. Takayama, E. Yashima, Angew. Chem. Int. Ed. 2020, 59, 14671-14676; Angew. Chem. 2020, 132, 14779-14784.

[9] G. Qin, Y. Wei, H. Kang, C. Dong, Anal. Methods. 2012, 4, 3928-3931.

[10] Y. Wei, W.-H. Chan, A. W. Lee, C. W. Huie, Chem. Commun. 2004, 288-289.

[11] X. Wu, C.-Y. Huang, D.-G. Chen, D. Liu, C. Wu, K.-J. Chou, B. Zhang, Y. Wang, Y. Liu, E. Y. Li, Nat. Commun. 2020, 11, 1-10.

[12] X. H. Zhang, Y. Wang, W. J. Jin, Anal. Chim. Acta. 2008, 622, 157-162.

[13] a) P. Sutter, S. Wimer, E. Sutter, Nature 2019, 570, 354-357; b) M. Singh, K. Liu, S. Qu, H. Ma, H. Shi, Z. An, W. Huang, Adv. Opt. Mater. 2021, 9, 2002197

[14] A. S. Carretero, A. S. Castillo, A. F. Gutiérrez, Crit. Rev. Anal. Chem. 2005, 35, 3-14.

[15] B. Chen, W. Huang, X. Nie, F. Liao, H. Miao, X. Zhang, G. Zhang, Angew. Chem. Int. Ed. 2021, 60, 16970-16973; Angew. Chem. 2021, 133, 17107-17110.

[16] Y. Lei, W. Dai, J. Guan, S. Guo, F. Ren, Y. Zhou, J. Shi, B. Tong, Z. Cai, J. Zheng, Angew. Chem. Int. Ed. 2020, 59, 16054-16060; Angew. Chem. 2020, 132, 16188-16194.

[17] K. Wen, S. Yu, Z. Huang, L. Chen, M. Xiao, X. Yu, L. Pu, J. Am. Chem Soc. 2015, 137, 4517-4524.

[18] L. Pu, Angew. Chem. Int. Ed. 2020, 59, 21814-21828; Angew. Chem. 2020, 132, 21998-22012.

[19] a) Y.-Y. Zhu, X.-D. Wu, S.-X. Gu, L. Pu, J. Am. Chem. Soc. 2018, 141, 175-181; b) F. Y. Thanzeel, A. Sripada, C. Wolf, J. Am. Chem. Soc. 2019, 141, 16382-16387.

[20] S.-L. Shi, Z. L. Wong, S. L. Buchwald, Nature 2016, 532, 353-356. 


\section{Entry for the Table of Contents}

Insert graphic for Table of Contents here.
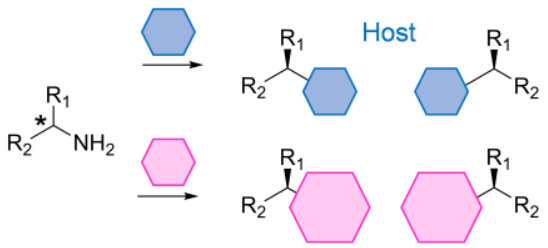

Guest

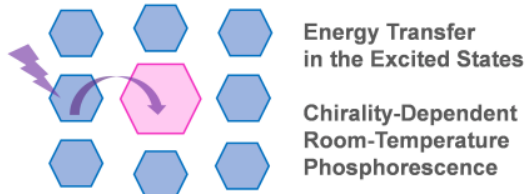

Guest-host systems exhibit highly enantioselective room-temperature phosphorescence enhancement of up to 196 folds and are applied to chiral recognition for chiral amino compounds with high sensitivity and ultratrace analysis. 